\title{
Strong laws for weighted sums of NA random variables
}

\section{Guang-hui Cai}

Received: 12 December 2005 / Published online: 4 December 2007

(C) The Author(s) 2007

\begin{abstract}
Strong laws are established for linear statistics that are weighted sums of an negatively associated (NA) random sample. The results obtained not only generalize the results of Sung (Stat. Probab. Lett. 52:413-419, 2001) to NA random variables, but also extend and sharpen them.
\end{abstract}

Keywords Almost sure convergence $\cdot$ Weighted sums $\cdot$ NA

\section{Introduction}

For negatively associated (NA) random variables: Joag and Proschan (1983) gives the following definition

Definition (Joag and Proschan 1983) A finite family of random variables $\left\{X_{i}, 1 \leq\right.$ $i \leq n\}$ is said to be negatively associated (NA) if for every pair of disjoint subsets, $T_{1}$ and $T_{2}$, of $\{1,2, \ldots, n\}$, we have

$$
\operatorname{Cov}\left(f_{1}\left(X_{i}, i \in T_{1}\right), f_{2}\left(X_{j}, j \in T_{2}\right)\right) \leq 0,
$$

whenever $f_{1}$ and $f_{2}$ are coordinatewise increasing and the covariance exists. An infinite family is negatively associated if every finite subfamily is negatively associated.

Let $\left\{X, X_{i}, i \geq 1\right\}$ be a sequence of i.i.d. random variables and $\left\{a_{n i}, 1 \leq i \leq\right.$ $n, n \geq 1\}$ be a triangular array of constants. The almost sure (a.s.) limiting behavior

Research supported by Youth Talent Foundation of Zhejiang Gongshang University (Q07-07).

G.-h. Cai $(\bowtie)$

Department of Statistics, Zhejiang Gongshang University, Hangzhou 310035,

People's Republic of China

e-mail: cghzju@163.com 
of weighted sums $\sum_{i=1}^{n} a_{n i} X_{i}$ has been studied by many authors (see, Sung 2001; Bai and Cheng 2000; Choi and Sung 1987; Cuzick 1995; Wu 1999). Recently Sung (2001) proved the following strong laws of large numbers:

Theorem A Let $\left\{X, X_{i}, i \geq 1\right\}$ be a sequence of i.i.d. random variables satisfying $E X=0$ and for any $h, \gamma>0, E \exp \left(h|X|^{\gamma}\right)<\infty$. And let $\left\{a_{n i}, 1 \leq i \leq n, n \geq 1\right\}$ be a triangular array of constants satisfying $A_{\alpha}=\limsup _{n \rightarrow \infty} A_{\alpha, n}<\infty, A_{\alpha, n}=$ $\sum_{i=1}^{n}\left|a_{n i}\right|^{\alpha} / n$ for some $1<\alpha \leq 2$. Then for $0<\gamma<1$ and $b_{n}=n^{\frac{1}{\alpha}}(\log n)^{\frac{1}{\gamma}}$

$$
\sum_{i=1}^{n} a_{n i} X_{i} / b_{n} \rightarrow 0 \text { a.s. }
$$

moreover, for $\gamma>1$ and $b_{n}=n^{\frac{1}{\alpha}}(\log n)^{\frac{1}{\gamma}+\delta}$

$$
\sum_{i=1}^{n} a_{n i} X_{i} / b_{n} \rightarrow 0 \text { a.s. }
$$

where $\delta=1-\frac{1}{\gamma}-\frac{\gamma-1}{1+\alpha \gamma-\alpha}$.

Theorem B Let $\left\{X, X_{i}, i \geq 1\right\}$ be a sequence of i.i.d. random variables satisfying $E X=0$ and for some $h, \gamma>0, E \exp \left(h|X|^{\gamma}\right)<\infty$. And let $\left\{a_{n i}, 1 \leq i \leq n, n \geq 1\right\}$ be a triangular array of constants satisfying $A_{\alpha}=\limsup _{n \rightarrow \infty} A_{\alpha, n}<\infty, A_{\alpha, n}=$ $\sum_{i=1}^{n}\left|a_{n i}\right|^{\alpha} / n$ for some $1<\alpha \leq 2$. Then for $0<\gamma \leq 1$ and $b_{n}=n^{\frac{1}{\alpha}}(\log n)^{\frac{1}{\gamma}+\beta}$ for $\beta>0$

$$
\sum_{i=1}^{n} a_{n i} X_{i} / b_{n} \rightarrow 0 \text { a.s. }
$$

moreover, for $\gamma>1$ and $b_{n}=n^{\frac{1}{\alpha}}(\log n)^{\frac{1}{\gamma}+\delta+\beta}$ for $\beta>0$

$$
\sum_{i=1}^{n} a_{n i} X_{i} / b_{n} \rightarrow 0 \text { a.s. }
$$

where $\delta=1-\frac{1}{\gamma}-\frac{\gamma-1}{1+\alpha \gamma-\alpha}$.

The main purpose of this paper is to establish the Marcinkiewicz-Zygmund strong laws for linear statistics of NA sequences of random variables. The results obtained not only generalize the results of Sung (2001) to NA random variables, but also extend and sharpen them.

\section{The Marcinkiewicz-Zygmund strong laws}

Throughout this paper, $C$ will represent a positive constant though its value may change from one appearance to the next, $a_{n}=O\left(b_{n}\right)$ will represent $a_{n} \leq C b_{n}$, and $a_{n} \ll b_{n}$ will represent $a_{n}=O\left(b_{n}\right)$. 
In order to prove our results, we need the following lemma and the concept of complete convergence. As for complete convergence, let $\left\{X, X_{n}, n \geq 1\right\}$ be a sequence of independent indentically distribution random variables (i.i.d) random variables and denote $S_{n}=\sum_{i=1}^{n} X_{i}$. The Hsu-Robbins-Erdös law of large numbers (Hsu and Robbins 1947; Erdös 1949) states that

$$
\forall \varepsilon>0, \quad \sum_{n=1}^{\infty} P\left(\left|S_{n}\right|>\varepsilon n\right)<\infty
$$

is equivalent to $E X=0$ and $E X^{2}<\infty$.

This is a foundamental theorm in probability theory and has been intensively investigated by many authors in the past decades. We can see in Petrov (1995), Chow and Teicher (1997) and Stout (1974). There have been many extensions in various directions for Hsu-Robbins-Erdös law of large numbers.

In order to prove our results, we need the following lemma.

Lemma 2.1 (Su et al. 1996; Shao 2000 or Yang 2000) Let $\left\{X_{i}, i \geq 1\right\}$ be a sequence of NA random variables, $E X_{i}=0, E\left|X_{i}\right|^{p}<\infty$ for some $p \geq 2$ and for every $i \geq 1$. Then there exists $C=C(p)$, such that

$$
E \max _{1 \leq k \leq n}\left|\sum_{i=1}^{k} X_{i}\right|^{p} \leq C\left\{\sum_{i=1}^{n} E\left|X_{i}\right|^{p}+\left(\sum_{i=1}^{n} E X_{i}^{2}\right)^{p / 2}\right\}
$$

The following is the main result in this paper.

Theorem 2.1 Let $\left\{X, X_{i}, i \geq 1\right\}$ be a sequence of $N A$ random variables with indentical distributions. And let $\left\{a_{n i}, 1 \leq i \leq n, n \geq 1\right\}$ be a triangular array of constants satisfying $\sum_{i=1}^{n}\left|a_{n i}\right|^{\alpha}=O(n)$ for $0<\alpha \leq 2$. Let $T_{n}=\sum_{i=1}^{n} a_{n i} X_{i}, n \geq 1$, $b_{n}=n^{\frac{1}{\alpha}}(\log n)^{\frac{1}{\gamma}} . E X=0$ when $1<\alpha \leq 2$. We assume that for some $h, \gamma>0$, $E \exp \left(h|X|^{\gamma}\right)<\infty$. Then

$$
\forall \varepsilon>0, \quad \sum_{n=1}^{\infty} n^{-1} P\left(\max _{1 \leq j \leq n}\left|T_{j}\right|>\varepsilon b_{n}\right)<\infty .
$$

Proof Without loss of generality, we can assume that $a_{n i} \geq 0$, for all $1 \leq i \leq n, n \geq$ 1. $\forall i \geq 1$, define $X_{i}^{(n)}=X_{i} I\left(\left|X_{i}\right| \leq b_{n}\right)+b_{n} I\left(X_{i}>b_{n}\right)-b_{n} I\left(X_{i}<-b_{n}\right)$, $T_{j}^{(n)}=\sum_{i=1}^{j}\left(a_{n i} X_{i}^{(n)}-E a_{n i} X_{i}^{(n)}\right)$, then $\forall \varepsilon>0$, 


$$
\begin{aligned}
& P\left(\max _{1 \leq j \leq n}\left|T_{j}\right|>\varepsilon b_{n}\right) \\
& \quad \leq P\left(\max _{1 \leq j \leq n}\left|X_{j}\right|>b_{n}\right)+P\left(\max _{1 \leq j \leq n}\left|T_{j}^{(n)}\right|>\varepsilon b_{n}-\max _{1 \leq j \leq n}\left|\sum_{i=1}^{j} E a_{n i} X_{i}^{(n)}\right|\right) .
\end{aligned}
$$

First we show that

$$
b_{n}^{-1} \max _{1 \leq j \leq n}\left|\sum_{i=1}^{j} E a_{n i} X_{i}^{(n)}\right| \rightarrow 0 \text {, as } n \rightarrow \infty .
$$

By $\sum_{i=1}^{n}\left|a_{n i}\right|^{\alpha}=O(n)$ and the Hölder inequality, $\forall 1 \leq k<\alpha$,

$$
\sum_{i=1}^{n}\left|a_{n i}\right|^{k} \leq\left(\sum_{i=1}^{n}\left|a_{n i}\right|^{k \frac{\alpha}{k}}\right)^{\frac{k}{\alpha}}\left(\sum_{i=1}^{n} 1\right)^{\frac{\alpha-k}{\alpha}} \leq C n .
$$

When $1<\alpha \leq 2$, using $E X=0$, (2.4), the Markov inequality and $E \exp \left(h|X|^{\gamma}\right)<$ $\infty$, as $n \rightarrow \infty$,

$$
\begin{aligned}
& b_{n}^{-1} \max _{1 \leq j \leq n}\left|\sum_{i=1}^{j} E a_{n i} X_{i}^{(n)}\right| \\
& \leq b_{n}^{-1} \sum_{i=1}^{n} E\left|a_{n i} X_{i}\right| I\left(\left|X_{i}\right|>b_{n}\right)+\sum_{i=1}^{n}\left|a_{n i}\right| P\left(\left|X_{i}\right|>b_{n}\right) \\
& \ll b_{n}^{-1} \sum_{i=1}^{n}\left|a_{n i}\right| E|X| I\left(|X|>b_{n}\right)+n P\left(|X|>b_{n}\right) \\
& \leq C b_{n}^{-1} n E|X| I\left(|X|>b_{n}\right)+n \frac{E \exp \left(h|X|^{\gamma}\right)}{\exp \left(h b_{n}^{\gamma}\right)} \\
& \ll C b_{n}^{-1} n \sum_{k=n}^{\infty} E|X| I\left(b_{k}<|X| \leq b_{k+1}\right)+n \exp \left(-h b_{n}^{\gamma}\right) \\
& \leq C b_{n}^{-1} n \sum_{k=n}^{\infty} b_{k+1} P\left(|X|>b_{k}\right)+n e^{-h n^{\gamma / \alpha} \log n} \\
& \leq C b_{n}^{-1} n \sum_{k=n}^{\infty} b_{k+1} \frac{E \exp \left(h|X|^{\gamma}\right)}{\exp \left(h b_{k}^{\gamma}\right)}+n^{1-h n^{\gamma / \alpha}} \\
& \leq C n^{-\frac{1}{\alpha}}(\log n)^{-\frac{1}{\gamma}} n n^{-1}+n^{1-h n^{\gamma / \alpha}} \\
& \leq C n^{-\frac{1}{\alpha}}(\log n)^{-\frac{1}{\gamma}}+n^{1-h n^{\gamma / \alpha}} \rightarrow 0 . \\
& \leq C b_{n}^{-1} n \sum_{k=n}^{\infty}(k+1)^{\frac{1}{\alpha}}(\log (k+1))^{\frac{1}{\gamma}} k^{-h k^{\gamma / \alpha}}+n^{1-h n^{\gamma / \alpha}} \\
& \leq
\end{aligned}
$$


By $\sum_{i=1}^{n}\left|a_{n i}\right|^{\alpha}=O(n)$ and the Hölder inequality, $\forall k \geq \alpha$, then

$$
\sum_{i=1}^{n}\left|a_{n i}\right|^{k}=\sum_{i=1}^{n}\left|a_{n i}\right|^{\alpha}\left|a_{n i}\right|^{k-\alpha} \leq C n n^{\frac{k-\alpha}{\alpha}}=C n^{\frac{k}{\alpha}}
$$

When $0<\alpha \leq 1$, using (2.6), the Markov inequality and $E \exp \left(h|X|^{\gamma}\right)<\infty$, as $n \rightarrow \infty$,

$$
\begin{aligned}
& b_{n}^{-1} \max _{1 \leq j \leq n}\left|\sum_{i=1}^{j} E a_{n i} X_{i}^{(n)}\right| \\
& \leq b_{n}^{-1} \sum_{i=1}^{n} E\left|a_{n i} X_{i}\right| I\left(\left|X_{i}\right| \leq b_{n}\right)+\sum_{i=1}^{n}\left|a_{n i}\right| P\left(\left|X_{i}\right|>b_{n}\right) \\
& \ll b_{n}^{-1} \sum_{i=1}^{n}\left|a_{n i}\right| E|X| I\left(|X| \leq b_{n}\right)+n^{1 / \alpha} P\left(|X|>b_{n}\right) \\
& \leq C b_{n}^{-1} n^{\frac{1}{\alpha}} E|X| I\left(|X| \leq b_{n}\right)+n^{1 / \alpha} \frac{E \exp \left(h|X|^{\gamma}\right)}{\exp \left(h b_{n}^{\gamma}\right)} \\
& =C b_{n}^{-1} n^{\frac{1}{\alpha}} \sum_{k=2}^{n} E|X| I\left(b_{k-1}<|X| \leq b_{k}\right)+n^{1 / \alpha} \exp \left(-h b_{n}^{\gamma}\right) \\
& \leq C b_{n}^{-1} n^{\frac{1}{\alpha}} \sum_{k=2}^{n} b_{k} P\left(|X|>b_{k-1}\right)+n^{1 / \alpha} e^{-h n^{\gamma / \alpha} \log n} \\
& \leq C b_{n}^{-1} n^{\frac{1}{\alpha}} \sum_{k=2}^{n} b_{k} \frac{E \exp \left(h|X|^{\gamma}\right)}{\exp \left(h b_{k-1}^{\gamma}\right)}+n^{\frac{1}{\alpha}-h n^{\gamma / \alpha}} \\
& \leq C b_{n}^{-1} n^{\frac{1}{\alpha}} \sum_{k=2}^{n} k^{\frac{1}{\alpha}}(\log k)^{\frac{1}{\gamma}}(k-1)^{-h(k-1)^{\gamma / \alpha}}+n^{\frac{1}{\alpha}-h n^{\gamma / \alpha}} \\
& \leq C(\log n)^{-\frac{1}{\alpha}}(\log n)^{-\frac{1}{\gamma}} n^{\frac{1}{\alpha}}+n^{\frac{1}{\alpha}-h n^{\gamma / \alpha}}-h n^{\gamma / \alpha} \rightarrow 0 . \\
& \leq
\end{aligned}
$$

(2.3) follows from (2.5) and (2.7).

From (2.2) and (2.3), it follows that for large enough $n$

$$
P\left(\max _{1 \leq j \leq n}\left|T_{j}\right|>\varepsilon b_{n}\right) \leq \sum_{j=1}^{n} P\left(\left|X_{j}\right|>b_{n}\right)+P\left(\max _{1 \leq j \leq n}\left|T_{j}^{(n)}\right|>\frac{\varepsilon}{2} b_{n}\right) .
$$


Hence we need only to prove that

$$
\begin{aligned}
& I=: \sum_{n=1}^{\infty} n^{-1} \sum_{j=1}^{n} P\left(\left|X_{j}\right|>b_{n}\right)<\infty, \\
& I I=: \sum_{n=1}^{\infty} n^{-1} P\left(\max _{1 \leq j \leq n}\left|T_{j}^{(n)}\right|>\frac{\varepsilon}{2} b_{n}\right)<\infty .
\end{aligned}
$$

From the fact that $E \exp \left(h|X|^{\gamma}\right)<\infty$, it follows easily that

$$
\begin{aligned}
I & =\sum_{n=1}^{\infty} n^{-1} n P\left(|X|>b_{n}\right) \\
& =\sum_{n=1}^{\infty} P\left(|X|>b_{n}\right) \\
& \leq \sum_{n=1}^{\infty} \frac{E \exp \left(h|X|^{\gamma}\right)}{\exp \left(h b_{n}^{\gamma}\right)} \\
& \leq C \sum_{n=1}^{\infty} \frac{1}{n^{h^{\gamma / \alpha}}}<\infty .
\end{aligned}
$$

By Lemma 2.1, it follows that

$$
\begin{aligned}
I I & \leq C \sum_{n=2}^{\infty} n^{-1} b_{n}^{-q} E \max _{1 \leq j \leq n}\left|T_{j}^{(n)}\right|^{q} \\
& \leq C \sum_{n=2}^{\infty} n^{-1} b_{n}^{-q}\left\{\sum_{j=1}^{n} E\left|a_{n j} X_{j}^{(n)}\right|^{q}+\left(\sum_{j=1}^{n} E\left|a_{n j} X_{j}^{(n)}\right|^{2}\right)^{q / 2}\right\} \\
& =: I I_{1}+I I_{2} .
\end{aligned}
$$

Let $\max (2, \alpha, \gamma+1) \leq q$, using (2.6), we have

$$
\begin{aligned}
I_{1} & =C \sum_{n=2}^{\infty} n^{-1} b_{n}^{-q}\left\{\sum_{i=1}^{n}\left|a_{n i}\right|^{q} E|X|^{q} I\left(|X| \leq b_{n}\right)+\sum_{i=1}^{n}\left|a_{n i}\right|^{q} P\left(|X|>b_{n}\right)\right\} \\
\leq & C \sum_{n=2}^{\infty} n^{-1} b_{n}^{-q}\left\{n^{\frac{q}{\alpha}} E|X|^{q} I\left(|X| \leq b_{n}\right)+n^{\frac{q}{\alpha}} P\left(|X|>b_{n}\right)\right\} \\
\leq & C \sum_{n=2}^{\infty} n^{-1} b_{n}^{-q} n^{\frac{q}{\alpha}} \sum_{k=2}^{n} E|X|^{q} I\left(b_{k-1}<|X| \leq b_{k}\right) \\
& +C \sum_{n=2}^{\infty} n^{-1} b_{n}^{-q} n^{\frac{q}{\alpha}} \frac{E \exp \left(h|X|^{\gamma}\right)}{\exp \left(h b_{n}^{\gamma}\right)}
\end{aligned}
$$




$$
\begin{aligned}
\leq & C \sum_{k=2}^{\infty} \sum_{n=k}^{\infty} n^{-1+\frac{q}{\alpha}} n^{-q / \alpha}(\log n)^{-q / \gamma} b_{k}^{q} P\left(|X|>b_{k-1}\right) \\
& +C \sum_{n=2}^{\infty} n^{-1}(\log n)^{-q / \gamma} n^{-h n^{\gamma / \alpha}} \\
\leq & C \sum_{k=2}^{\infty} b_{k}^{q} \frac{E \exp \left(h|X|^{\gamma}\right)}{\exp \left(h b_{k-1}^{\gamma}\right)}+C \sum_{n=2}^{\infty} n^{-1}(\log n)^{-q / \gamma} n^{-h n^{\gamma / \alpha}} \\
\leq & C \sum_{k=2}^{\infty} k^{\frac{q}{\alpha}}(\log k)^{\frac{q}{\gamma}}(k-1)^{-h(k-1)^{\gamma / \alpha}} \\
& +C \sum_{n=2}^{\infty} n^{-1}(\log n)^{-q / \gamma} n^{-h n^{\gamma / \alpha}}<\infty .
\end{aligned}
$$

By $0<\alpha \leq 2,(2.6)$ and $q \geq \max \{2, \gamma+1\}$, we have

$$
\begin{aligned}
I I_{2}= & C \sum_{n=2}^{\infty} n^{-1} b_{n}^{-q}\left\{\left(\sum_{i=1}^{n}\left|a_{n i}\right|^{2}\right)^{q / 2}\left(E|X|^{2} I\left(|X| \leq b_{n}\right)\right)^{q / 2}\right. \\
& \left.+\left(\sum_{i=1}^{n}\left|a_{n i}\right|^{2} P\left(|X|>b_{n}\right)\right)^{q / 2}\right\} \\
\leq & C \sum_{n=2}^{\infty} n^{-1} b_{n}^{-q}\left\{\left(n^{\frac{2}{\alpha}}\right)^{q / 2}\left(E|X|^{2} I\left(|X| \leq b_{n}\right)\right)^{q / 2}+\left(n^{\frac{2}{\alpha}}\right)^{q / 2} P\left(|X|>b_{n}\right)\right\} \\
= & C \sum_{n=2}^{\infty} n^{-1}(\log n)^{-q / \gamma}\left(\sum_{k=2}^{n} E|X|^{2} I\left(b_{k-1}<|X| \leq b_{k}\right)\right)^{q / 2} \\
& +C \sum_{n=2}^{\infty} n^{-1}(\log n)^{-q / \gamma} P\left(|X|>b_{n}\right) \\
\leq & C \sum_{n=2}^{\infty} n^{-1}(\log n)^{-q / \gamma}\left(\sum_{k=2}^{n} b_{k}^{2} P\left(|X|>b_{k-1}\right)\right)^{q / 2} \\
& +C \sum_{n=2}^{\infty} n^{-1}(\log n)^{-q / \gamma} \frac{E \exp \left(h|X|^{\gamma}\right)}{\exp \left(h b_{n}^{\gamma}\right)} \\
\leq & C \sum_{n=2}^{\infty} n^{-1}(\log n)^{-q / \gamma}\left(\sum_{k=2}^{n} b_{k}^{2} \frac{E \exp \left(h|X|^{\gamma}\right)}{\exp \left(h b_{k-1}^{\gamma}\right)}\right)^{q / 2} \\
+ & C \sum_{n=2}^{\infty} n^{-1}(\log n)^{-q / \gamma} n^{-h n^{\gamma / \alpha}} \\
& \\
&
\end{aligned}
$$




$$
\begin{aligned}
\leq & C \sum_{n=2}^{\infty} n^{-1}(\log n)^{-q / \gamma}\left(\sum_{k=2}^{n} \frac{k^{\frac{2}{\alpha}}(\log k)^{\frac{2}{\gamma}}}{\exp \left(h(k-1)^{\gamma / \alpha} \log (k-1)\right)}\right)^{q / 2} \\
& +C \sum_{n=2}^{\infty} n^{-1}(\log n)^{-q / \gamma} n^{-h n^{\gamma / \alpha}} \\
= & C \sum_{n=2}^{\infty} n^{-1}(\log n)^{-q / \gamma}\left(\sum_{k=2}^{n} k^{\frac{2}{\alpha}}(\log k)^{\frac{2}{\gamma}}(k-1)^{-h(k-1)^{\gamma / \alpha}}\right)^{q / 2} \\
& +C \sum_{n=2}^{\infty} n^{-1}(\log n)^{-q / \gamma} n^{-h n^{\gamma / \alpha}} \\
\leq & C \sum_{n=2}^{\infty} n^{-1}(\log n)^{-q / \gamma}\left(\sum_{k=2}^{n} k^{-2}\right)^{q / 2}+C \sum_{n=2}^{\infty} n^{-1}(\log n)^{-q / \gamma} n^{-h n^{\gamma / \alpha}} \\
\leq & C \sum_{n=2}^{\infty} n^{-1}(\log n)^{-q / \gamma}+C \sum_{n=2}^{\infty} n^{-1}(\log n)^{-q / \gamma} n^{-h n^{\gamma / \alpha}}<\infty
\end{aligned}
$$

Putting (2.11) and (2.12) into (2.10) yields $I I<\infty$. Now we complete the proof of Theorem 2.1.

Corollary 2.1 Under the conditions of Theorem 2.1,

$$
\lim _{n \rightarrow \infty} \frac{\left|T_{n}\right|}{b_{n}}=0 \text { a.s. }
$$

Proof By (2.1), we have

$$
\begin{aligned}
\infty & >\sum_{n=1}^{\infty} n^{-1} P\left(\max _{1 \leq j \leq n}\left|T_{j}\right|>\varepsilon b_{n}\right) \\
& =\sum_{i=0}^{\infty} \sum_{n=2^{i}}^{2^{i+1}-1} n^{-1} P\left(\max _{1 \leq j \leq n}\left|T_{j}\right|>\varepsilon n^{\frac{1}{\alpha}}(\log n)^{\frac{1}{\gamma}}\right) \\
& \geq \frac{1}{2} \sum_{i=1}^{\infty} P\left(\max _{1 \leq j \leq 2^{i}}\left|T_{j}\right|>\varepsilon 2^{\frac{i+1}{\alpha}}\left(\log 2^{i+1}\right)^{\frac{1}{\gamma}}\right) .
\end{aligned}
$$

By the Borel-Cantelli Lemma, we have

$$
P\left(\max _{1 \leq j \leq 2^{i}}\left|T_{j}\right|>\varepsilon 2^{\frac{i+1}{\alpha}}\left(\log 2^{i+1}\right)^{\frac{1}{\gamma}} \text {, i.o. }\right)=0 .
$$


Hence

$$
\lim _{i \rightarrow \infty} \frac{\max _{1 \leq j \leq 2^{i}}\left|T_{j}\right|}{2^{\frac{i+1}{\alpha}}\left(\log 2^{i+1}\right)^{\frac{1}{\gamma}}}=0 \text { a.s. }
$$

and using

$$
\max _{2^{i-1} \leq n<2^{i}} \frac{\left|T_{n}\right|}{b_{n}} \leq 2^{\frac{2}{\alpha}} \frac{\max _{1 \leq j \leq 2^{i}}\left|T_{j}\right|}{2^{\frac{i+1}{\alpha}}\left(\log 2^{i+1}\right)^{\frac{1}{\gamma}}}\left(\frac{i+1}{i-1}\right)^{\frac{1}{\gamma}}
$$

we have

$$
\lim _{n \rightarrow \infty} \frac{\left|T_{n}\right|}{b_{n}}=0 \text { a.s. }
$$

Remark Corollary 2.1 not only generalizes the results of Sung (2001) to NA random variables, but also extends and sharpens them.

Acknowledgments The author would like to thank the anonymous referee for his/her valuable comments.

Open Access This article is distributed under the terms of the Creative Commons Attribution Noncommercial License which permits any noncommercial use, distribution, and reproduction in any medium, provided the original author(s) and source are credited.

\section{References}

Bai ZD, Cheng PE (2000) Marcinkiewicz strong laws for linear statistics. Stat Probab Lett 46:105-112

Choi BD, Sung SH (1987) Almost sure convergence theorems of weighted sums of random variables. Stoch Anal Appl 5:365-377

Chow YS, Teicher H (1997) Probability theory: independence, interchangeability, martingales, 3rd edn. Springer, New York

Cuzick J (1995) A strong law for weighted sums of i.i.d. random variables. J Theor Probab 8:625-641

Erdös P (1949) On a theorem of Hsu-Robbins. Ann Math Stat 20:286-291

Hsu PL, Robbins H (1947) Complete convergence and the law of larege numbers. Proc Natl Acad Sci USA 33(2):25-31

Joag DK, Proschan F (1983) Negative associated of random variables with application. Ann Stat 11:286295

Petrov VV (1995) Limit theorems of probability theory sequences of independent random variables. Oxford Science Publications, Oxford

Shao QM (2000) A comparison theorem on moment inequalities between Negatively associated and independent random variables. J Theor Probab 13:343-356

Stout W (1974) Almost sure convergence. Academic, New York

Su C, Zhao LC, Wang YB (1996) Moment inequalities and weak convergence for NA sequences. Sci China Ser A 26:1091-1099

Sung SH (2001) Strong laws for weighted sums of i.i.d. random variables. Stat Probab Lett 52:413-419

Wu WB (1999) On the strong convergence of a weighted sums. Stat Probab Lett 44:19-22

Yang SC (2000) Moment inequality of random variables partial sums. Sci Chin Ser A 30:218-223 